\title{
Hot Stamped Conductors on Nano-Micro Wires Made by Electroplating for Medical Sensors and Micro-Implants
}

\author{
Dipl.-Ing. Tim Rossner ${ }^{1}$, Dipl.-Ing. Alexandra Lyashenko², Prof. Dr.-Ing. Edgar Dörsam², Reza Sarwar, \\ M.Sc. ${ }^{-1}$, Prof. Dr.-Ing. habil. Roland Werthschützky ${ }^{1}$ \\ ${ }^{1}$ Institut für Elektromechanische Konstruktionen, Technische Universität Darmstadt, Merckstraße 25, \\ 64283 Darmstadt, Germany \\ ${ }^{2}$ Institut für Druckmaschinen und Druckverfahren, Technische Universität Darmstadt, \\ Magdalenenstraße 2, 64289 Darmstadt, Germany \\ t.rossner@emk.tu-darmstadt.de
}

\begin{abstract}
This paper presents a novel concept of hot stamped conductors on wires in a nano- or microscale. The process of producing arrays of wires selectively on the desired contact pad by electroplating of a template foil is described. The new concept provides electrical conductivity by penetration of wire arrays through the conductive layer of a hot stamping foil. Because of the small cross section of the wires, a large surface provides a low resistance between the contact pad and the conductor. With this process the non-conductive layer of the hot stamping foil does not have to be removed. The wires are flexible and provide a good alternative for electrical connection of flexible electronics. Also the process temperature is low, which makes it possible to use materials which are sensitive to high temperatures. When renouncing the use of temperature sensitive materials, this method can be applicable for high temperature operation due to the lack of adhesive or solder.
\end{abstract}

Key words: Nanowire, microwire, hot stamping, hot stamped conductors, functional printing, electroplating, electrical contact.

\section{Introduction}

The demand of further miniaturized systems for medical devices is growing due to demographic change. The percentage of minimally invasive operations is increasing, so a high demand of smaller and more complex systems for medical applications can be expected. Electrical contacts are a key technology for reliable operation of microsystems. In the course of miniaturization, new technologies for conductors and functional structures are necessary.

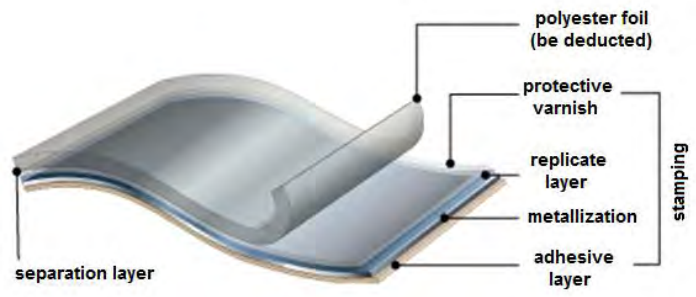

Figure 1: Layer composition of a hot stamping foil [1]

At the Institute of Printing Science and Technology of Technische Universität Darmstadt printing of electronic components and devices is investigated. One of these methods is hot stamping technology. For this process, so-called hot stamping foils are used. The basic layer of a hot stamping foil is a 12 micron polyester foil on which an approximately 0.01 micron separation layer is applied. On the separation layer, three layers are deposited: a protective varnish layer of 1.0 - 1.5 microns in thickness, a $0.02-0.1$ micron thick metal layer, at last, an adhesive layer [2]. In most cases for the metal layer aluminum is used [3] (Figure 1).

The hot stamping foils can be used for applications as strip conductors or functional devices such as strain gauges (Figure 2) and are transferred by heat and pressure of a hot stamp to the substrate. The non-conductive adhesive layer is heat activated and allows fixation on other layers on the substrate. Afterwards the polyester foil is removed.

Due to the low thickness of the metal layer and by the use of high-temperature-sensitive layers for the adhesive and protective varnish, common methods such as soldering or microwelding are not suitable for connecting these foils to devices or sensors. Until now, printed layers are electrically contacted by using 
isotropic conductive adhesive [4]. The conductive layers of hot stamped foils are always covered with a non-conductive protective varnish so it cannot be electrically connected directly with high-temperature technologies or adhesives.

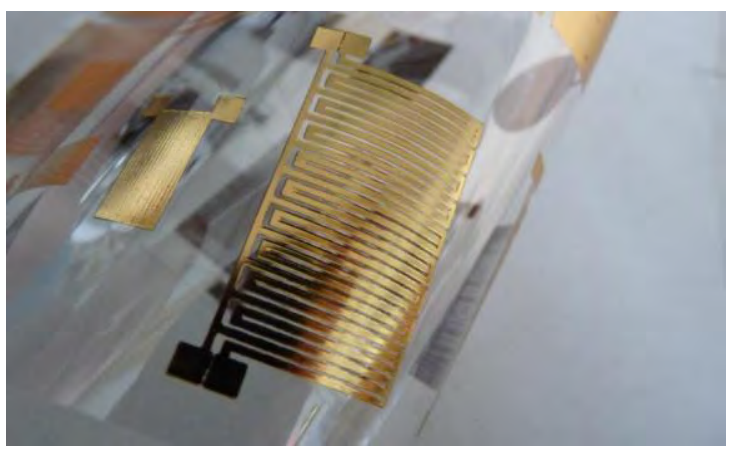

Figure 2: Hot stamped strain gauge sensor

In this paper, a novel approach is investigated to use electrodeposited wires of nanoscale or within a few microns in diameter to easily provide conductivity to silicon chips or flexible strips at the process of hot stamping. Due to the small cross section of the wires and a very high aspect ratio, these nano or micro wire arrays have a very large surface to volume ratio. This promises a low contact resistant when using these wires for electrical contacts. Another advantage of using small wires is the flexibility, because of the small distance to the neutral phase when bended.

Nanowires are investigated in several applications such as acceleration sensor [5], gas flow sensor [5] or humidity micro switch [6]. This new approach provides permanent electrical conductivity by penetrating the conductive layer of hot stamping foils with nanowires. The processes are described in the following.

\section{Electroforming of nanowires}

The production of wire arrays is made by electroplating of template foils. The process flow is shown in Figure 3 and described below.

First, the desired substrate is to be structured. In this case a 125 micron Hostaphan PET foil is used. This foil is coated with chrome and nickel by vaporizing (PVD). A photolithographic process is used to provide conductors und contact pads. Another photolithographic process with another mask is used to cover all conductors and areas which should not be covered with wires.

For the production of wire arrays, a template foil is used. A polycarbonate foil is irradiated with high energy heavy ions. Every single ion produces defects in the foil, which can be opened by a selective wet chemical etching to cylindrical capillaries from $10 \mathrm{~nm}$ to several microns. The density of these pinholes can also be adjusted.

a)

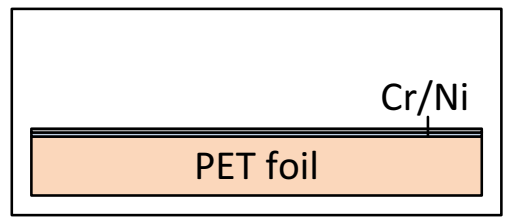

b)

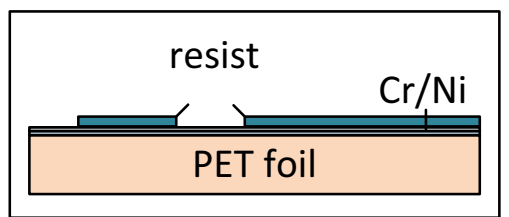

c)

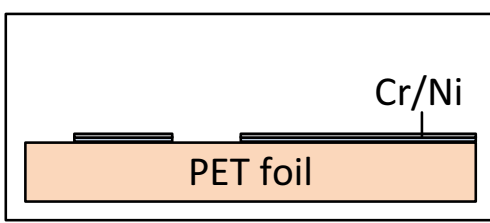

d)

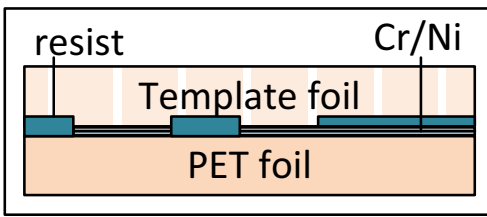

e)

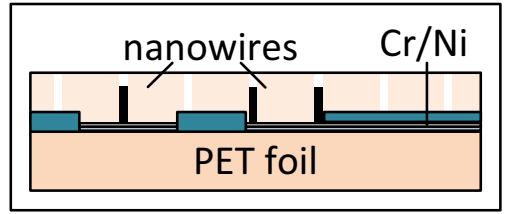

f)

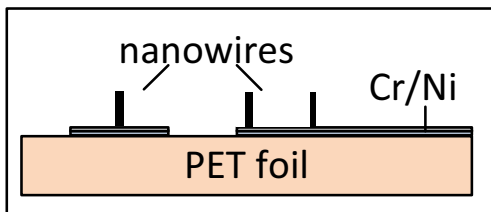

Figure 3: Fabrication of wire arrays: a) Vaporization (PVD) of chrome and nickel on PET substrate, b) photolithographic resist structured, c) etching of metallic layers and removal of resist d) masking of contact pads and lamination of template foil e) growth of nanowires by electroplating f) removal of template foil and resist by solvent.

In order to get wires, the capillaries have to be filled. To do this, the template foil is laminated onto the structured substrate mentioned above. A galvanic process fills the channels of the template foil with nickel, copper, or gold depending of the electrolytic fluid used. In order to make sure all pinholes are filled with electrolytic fluid, the galvanic chamber can be evacuated. This process can be necessary for wires with diameters of $\mathrm{nm}$ because of trapped air in the capillaries. The galvanic deposition is done potentiostatically with a 2 or 3 electrodes 
arrangement on a hotplate to control temperature. Detailed information about the process is given in [5] and [7].

The length of wires depends on the current density and time of galvanic deposition. The length must not exceed the thickness of the template foil (overgrowth) to provide an array of single wires instead of a connected array of wires by a solid plate on the top. After the electrolytic process, the template foil can be removed by solvent or oxygen plasma. The prepared wires can be enhanced by a chemical coating, for example with gold, in order to reduce contact resistance.

Figure 4 shows a picture of an array of nanowires. It shows the hard line between the structured area with nanowires and the nonconductive substrate. It also shows the high aspect ratio of the wires.

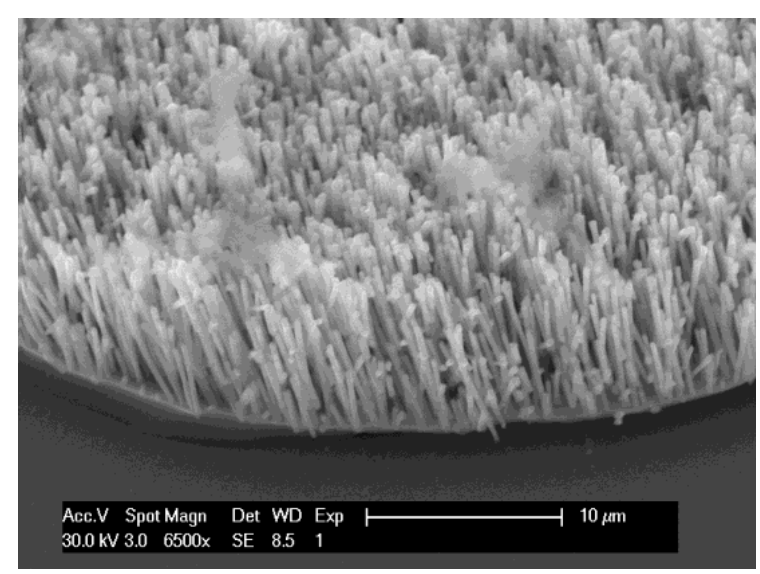

Figure 4: REM image of a nanowire array

\section{Concept}

The novel method for contacting thin hot stamped layers provides electrical conductivity by penetration of the metallic layer by nano or micro wires. Because of the selective process of electroplating, the wire arrays only grow on areas that are electrically connected to the electrode. Silicon chips usually provide a passivation of conductors and other metallic surfaces except the contact pads. Therefore a lithographic process is not needed in most cases when using silicon chips instead of the test structure mentioned above.

The length of the wires $\left(I_{\text {wire }}\right)$ has to be chosen, so the thickness of the non-conductive adhesive layer $\left(d_{N C L}\right)$, as well as the thickness of the conductive metal layer $\left(d_{c L}\right)$ can be penetrated, but the protective varnish layer $\left(d_{P V L}\right)$ is not exceeded completely as shown in equation (1).

$\mathrm{d}_{\mathrm{NCL}}+\mathrm{d}_{\mathrm{CL}}<\mathrm{I}_{\text {wire }}<\mathrm{d}_{\mathrm{NCL}}+\mathrm{d}_{\mathrm{CL}}+\mathrm{d}_{\mathrm{PVL}}$ (1)
After the wire arrays are formed on the contact surfaces, electrical contact is produced selectively in the process of hot stamping. Figure 5 shows the process for providing electrical conductivity between a silicon sensor and a hot stamping foil used for example as conductor strips.

First there is a lamination of the silicon chip into a substrate, to provide a flat surface. Wires are made only on the contact pads by electroplating with a template foil as described in the paragraph above. The stamping of the hot stamping foil is done by a heat sealing press at temperatures of $120^{\circ}$ Celsius and controlled pressure. Figure 6 shows the setup of hot stamping. The force while hot stamping has to be exactly vertical to the surface of the chip. Otherwise crippling of the wires can occur instead of penetration of the foil.

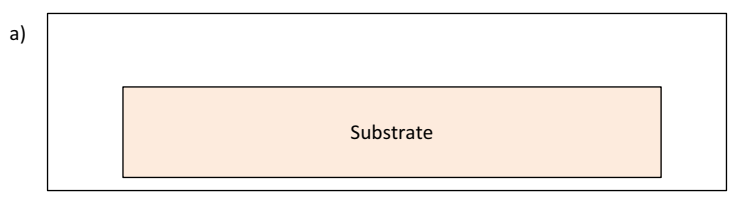

b)

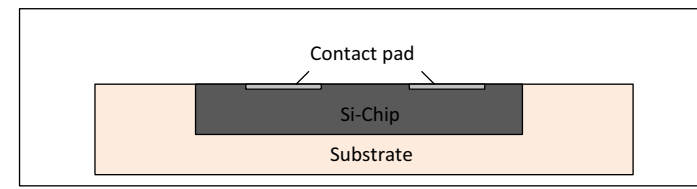

c)
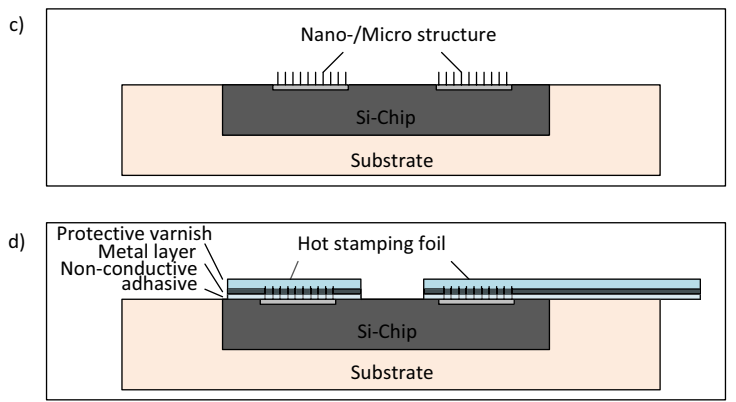

Figure 5: Concept of conductors hot stamped on wire arrays made by electroplating: a) Flexible substrate suitable for lamination, b) lamination of silicon chip into substrate, c) growth of nanowires on contact pads, d) stamping of hot stamping foil with heat sealing press.

The structured hot stamp foils can be used as a conductor strip between a sensor and electronics or the power source in this example. By using ultra-thin chips the arrangement can be bendable. This new approach does not stiffen the contact area as much as most technologies at the state of the art do. Because the wires can move relatively to each other, the contact area can absorb mechanical stress and is suitable for flexible electronics. This can be a big advantage for applications in flexible implants. Another benefit of the new technology is the lack of harmful substances as used in 
solder or electrically conductive adhesives which makes it predestined for medical application.

The process temperature of $120^{\circ}$ Celsius or even lower is applicable for most scenarios.

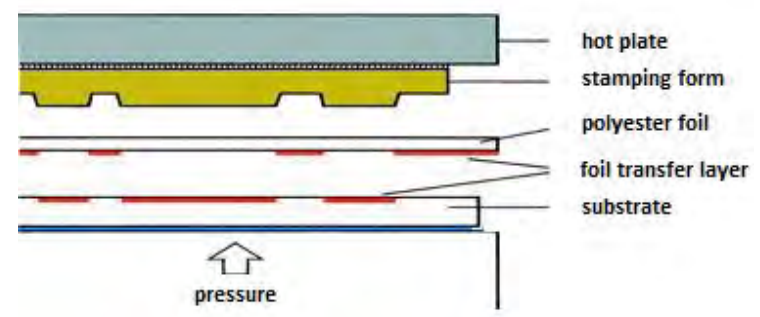

Figure 6: Setup of a hot stamping technology

\section{Conclusion}

This paper shows a concept of a novel approach to use nano- or microwires for electrical connection between contact pads and very thin hot stamping foils. The process of growing nanowires by electroplating of template foils is shown as well as a process to apply the hot stamping foil on silicon chips. The processes are under investigation and optimization on lithographic processed test structures and results will follow soon.

This new approach has some major advantages. First it is a low-temperature process at about 120 degrees Celsius. This allows the use of temperature-sensitive materials.

Because of the high surface of the nanowires, the conductivity is expected to be very high, although the metal layer is penetrated through a non-conductive layer. The hot stamping foil does not need to be structured to provide blank metal layers.

The nanowires are not mechanically connected to each other. So the electrical contact does not reinforce the structure in a way, soldering or adhesive bonding does. Using ultrathin hot stamping foils of a thickness less than 2 microns, combined with this novel approach to use nanowires for electrical contact in combination with ultrathin electronics, has a huge potential for flexible electronics. With this technology hot stamp foils can be used for many devices - conductors or functional structures such as strain gauges.

The connection between nanowires and the conductive metal layer does not require additional adhesives or other harmful additives. Under higher pressure a ductile deformation of the wires and the metal layer can provide good mechanical properties. This fact can be a key to biocompatible electrical connection for medical applications.

Especially the last two advantages makes this novel approach predestined for flexible implants in the human body. Further investigations on long-term stability and mechanical and electrical properties are planned.

It is to be investigated if this process is suitable for high temperature applications. This novel approach provides conductivity by penetration of metal wires on a metal foil. If it is possible to do plastic deformation and to provide a passivation that is capable of high temperatures, this novel approach could be used for operation temperatures over $300^{\circ}$ Celsius.

\section{References}

[1] LEONHARD KURZ Stiftung \& Co.KG online, http://www.kurz.de/de/ from 31.01.2013.

[2] Arbeitskreis Praegefoliendruck e.V., Praegefoliendruck: Verfahren, Technik und Gestaltung; ISBN 3-7785-2968-4, Huethig Verlag, Heidelberg, 2005.

[3] A. Lyashenko, L. Salun, E. Dörsam: Hot Stamping Technology For Functional Printing. In: 39th International Research Conference of iarigai, September 9-12, 2012, Ljubljana, Slovenia.

[4] A. Lyashenko, L. Salun, E. Dörsam, Contacting method for printed electronics. In: Large-area, Organic \& Printed Electronics Convention (LOPE-C), June 19-21, Munich, Germany.

[5] F. Greiner, S. Quednau, F. Dassinger, R. Sarwar, H. F. Schlaak, M. Guttmann, P. Meyer, Fabrication techniques for multiscale 3D-MEMS with vertical metal micro- and nanowire integration.

In: IOP Science Journal of Micromechanics and Microengineering, 14-Sep-12

[6] C. Bellmann, R. Sarwar, A. Steinke, T. Frank, H. F. Schlaak, G. Gerlach, Development of a humidity micro switch based on humiditysensitive polymers.

In: 23rd MME of the IImenau University of Technology, Germany, Paper number 1631, 1517/09/2012

[7] F. Dassinger, S. Quednau, F. Greiner, H. F. Schlaak, M. Hottes, C. Stegmann, M. Rauber, W. Ensinger, Application of integrated nano structures in micro systems. In: Mikro-Nano-Integration. VDE Verlag, Berlin, Germany, Jan. 2013 